\title{
Tristability in Viscoelastic Flow Past Side-by-Side Microcylinders
}

\author{
Cameron C. Hopkins ๑, Simon J. Haward@, and Amy Q. Shen® \\ Okinawa Institute of Science and Technology Graduate University, Onna-son, Okinawa 904-0495, Japan
}

(Received 16 October 2020; accepted 4 January 2021; published 1 February 2021)

\begin{abstract}
Viscoelastic flows through microscale porous arrays exhibit complex path selection and switching phenomena. However, understanding this process is limited by a lack of studies linking between a single object and large arrays. Here, we report experiments on viscoelastic flow past side-by-side microcylinders with variable intercylinder gap. With increasing flow rate, a sequence of two imperfect symmetry-breaking bifurcations forces selection of either one or two of the three possible flow paths around the cylinders. Tuning the gap length through the value where the first bifurcation becomes perfect reveals regions of bistability and tristability in a dimensionless flow rate-gap length phase diagram.
\end{abstract}

DOI: 10.1103/PhysRevLett.126.054501

Since the advent of microfluidics in the early 2000s [1,2], geometries with length scales $\ell \sim O(100 \mu \mathrm{m})$ have become a vital tool in experimental fluid dynamics. At the microscale, viscoelastic fluids (with properties between viscous liquids and elastic solids) can flow with negligible inertia (Reynolds number $\operatorname{Re} \sim \ell \ll 1$ ), but high elasticity (Weissenberg number $\mathrm{Wi} \sim \ell^{-1} \gg 1$ ) [2]. In such flows, elasticity becomes the dominant source of nonlinearity, leading to instabilities [3-11], and time dependency that impact widespread processes ranging from jet fragmentation $[12,13]$ to hemodynamics $[14,15]$ and porous media flows [16-22]. Earlier studies of viscoelastic porous media flows focused on understanding the anomalous increase in bulk pressure drop with flow rate in terms of the extensional viscosity induced by squeezing and stretching components in the flow field [23-26]. However, more recent pore-scale visualization highlights the crucial role of elastic instability that results in complex path selection and switching phenomena now considered of fundamental importance in processes including enhanced oil recovery, groundwater remediation, filtration, and drug delivery [16-21].

Porous media are frequently modeled by ordered and disordered arrays of microfluidic circular cylinders [17-21, 27]. Flow past a single circular cylinder in a channel is an archetypal problem in fluid dynamics, and a "benchmark" for studying viscoelastic flows. The stagnation point downstream of a cylinder is a location where streamline curvature combines with strong velocity gradients, conditions that render viscoelastic base flows prone to linear instability [28-32]. Note that this is in contrast to the

Published by the American Physical Society under the terms of the Creative Commons Attribution 4.0 International license. Further distribution of this work must maintain attribution to the author(s) and the published article's title, journal citation, and DOI. nonlinear, subcritical instabilities that lead to elastic turbulence in parallel shear flows [30,31,33-36]. Downstream of a cylinder, for viscoelastic fluids with a shear-ratedependent viscosity (i.e., shear thinning), fluctuations caused by the perturbation to the base flow can lead to a supercritical bifurcation. This time-steady symmetrybreaking transition is characterized by the flow selecting a preferred path around one side of the cylinder or the other $[6-8,37]$. This behavior has clear relevance to understanding transport through porous arrays, but the interaction with neighboring array elements is lacking. Building "bottomup" complexity toward more realistic model systems, it is natural to consider two cylindrical objects either aligned in the flow direction or positioned side by side in a channel. Viscoelastic flow past two (or more) objects aligned on the flow axis is a well-studied problem (see, e.g., Refs. [9,31,38-40]). However, although equally important, the case of two objects positioned transverse to the flow has received scant attention, with only one numerical study conducted at high Reynolds number [41]. To date, creeping viscoelastic flow past side-by-side cylinders has not been studied.

In this Letter, we present microfluidic experiments of a viscoelastic shear-thinning fluid flowing past two microcylinders transverse to the primary flow direction (Fig. 1)

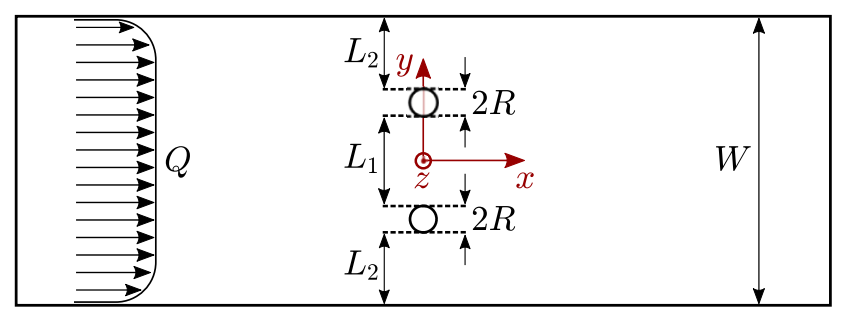

FIG. 1. Schematic diagram of the $x-y$ plane of the microfluidic channels $(W=400 \mu \mathrm{m}, R=20 \mu \mathrm{m}$ ). Flow is left to right at volumetric rate $Q$. 
and show that the resulting nonlinear flow behavior at high Wi is significantly influenced by the spacing of the cylinders. We show that, due to a combination of supercritical bifurcations that occur as $\mathrm{Wi}$ is varied, multiple stable time-steady flow states are possible in a given geometry. This is the first study of low-Re viscoelastic flow in such a geometry and serves as a fundamental contribution toward understanding deterministic path selection in porous media flow.

The model viscoelastic fluid is a well-studied aqueous wormlike micellar (WLM) solution consisting of $100 \mathrm{mM}$ cetylpyridinium chloride $(\mathrm{CPyCl})$ and $60 \mathrm{mM}$ sodium salicylate (NaSal) $[42,43]$. At $24^{\circ} \mathrm{C}$ (ambient laboratory temperature), the entangled WLM solution has a zero shear viscosity $\eta_{0}=47 \mathrm{Pas}$, exhibits a stress plateau (shearbanding region [44]), and in small-amplitude oscillation is well described by a single-mode Maxwell model with relaxation time $\lambda=1.7 \mathrm{~s}$ (Fig. S1 [45]).

Microfluidic channels (Fig. 1) were fabricated in fused silica by selective laser-induced etching [48]. The 11 channels used all have a rectangular cross section with width $W=400 \mu \mathrm{m}$ transverse to the flow (y direction), height $H=2000 \mu \mathrm{m}$ in the neutral $(z)$ direction, and length $25 \mathrm{~mm}$ in the primary flow $(x)$ direction. Each channel contains two cylinders of radius $R=20 \mu \mathrm{m}$ located halfway along the channel and equally spaced on either side of the $x$ axis. The intercylinder separation $L_{1}$ is varied between channels in the range $107<L_{1}<147 \mu \mathrm{m}$. The spacing between the cylinders and the channel sidewalls is $L_{2}=\left(W-L_{1}-4 R\right) / 2$, and we define a dimensionless gap ratio $G=L_{1} /\left(L_{1}+L_{2}\right)$. This parameter in principle spans $0<G<1$, where $G=0$ implies the two cylinders are touching at the channel centerline, while $G=1$ implies the cylinders are touching opposite channel walls. The channels used span $0.50 \leq G \leq 0.62$, encompassing the full range of flow behavior.

Flow is driven by syringe pumps (Cetoni $\mathrm{GmbH})$ programmed to impose quasistatic variations in the volumetric flow rate $Q$; hence average flow velocity $U=Q / W H$ and Weissenberg number $\mathrm{Wi}=\lambda U / R$. Quantitative spatially resolved flow fields are obtained using microparticle image velocimetry (TSI Inc., [49,50]). At each imposed $\mathrm{Wi}$, the motion of a low concentration of fluorescent seeding particles ( $2 \mu \mathrm{m}$ diameter Fluoromax red, Thermo Scientific Inc.) is captured at the channel half-height ( $z=0$ plane) using an inverted microscope (Nikon Ti) with a $5 \times, \mathrm{NA}=$ 0.15 numerical aperture objective lens and a high speed camera (Phantom Miro) working in frame-straddling mode at $25 \mathrm{~Hz}$. Cross-correlation between images yields velocity vectors $\mathbf{u}=(u, v)$. Since the flows examined are all time invariant, data are ensemble averaged over a $6 \mathrm{~s}$ sampling window. The shear-thinning nature of the WLM solution renders the flow profile essentially pluglike over most of the channel cross section [7]. Therefore, the shear rate

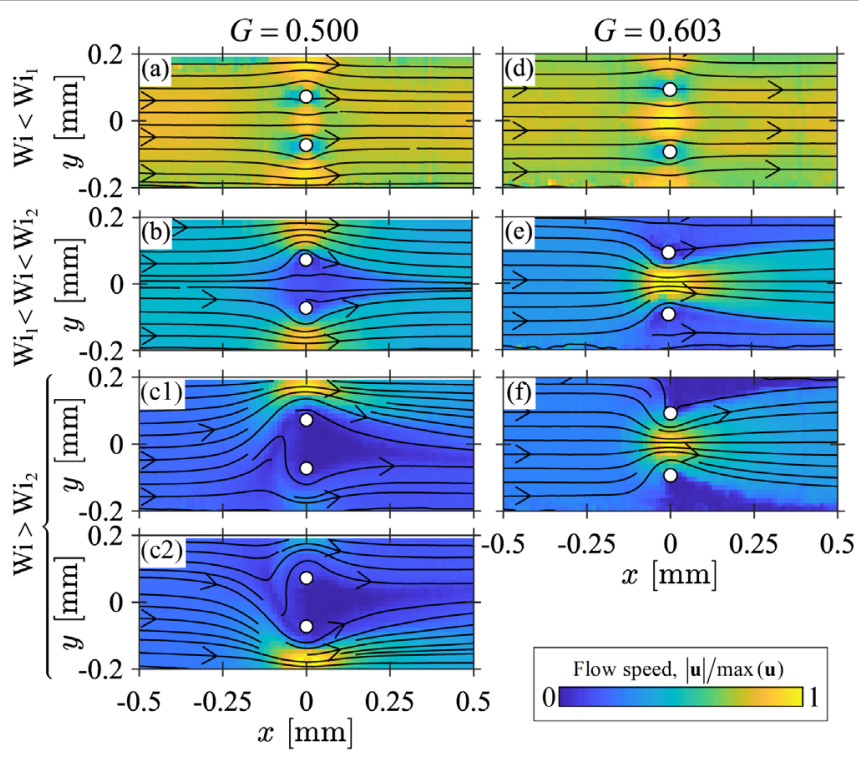

FIG. 2. Evolution of velocity fields with Wi for the WLM solution in channels with $G=0.500$ (a)-(c) and $G=0.603$ (d)(f). Panels ( $c 1)$ and ( $c 2)$ indicate the two possible states for $G=$ 0.500 and $\mathrm{Wi}>\mathrm{Wi}_{2}$.

near the cylinders is small and we define $\operatorname{Re}=\rho U R / \eta_{0}$, where $\rho=1000 \mathrm{~kg} \mathrm{~m}^{-3}$ is the fluid density. In all experiments, $\operatorname{Re} \lesssim 10^{-4}$.

Flow fields representative of those observed as Wi is varied are shown in Fig. 2 using two channels with contrasting $G$. Figures 2(a)-2(c) and Figs. 2(d)-2(f) illustrate the behavior for "small" and "large" $G$, respectively. Irrespective of $G$, for low $\mathrm{Wi}<\mathrm{Wi}_{1} \approx 15$ [Figs. 2(a) and 2(d)], elastic and inertial forces are small and the flow is dominated by the viscous force. Flow is approximately symmetric about $x=0$ and $y=0$, and fluid passes through all three available gaps. For small $G=0.500$, as Wi exceeds $\mathrm{Wi}_{1}$ [Fig. 2(b)], elasticity dominates and the system undergoes a first transition from the low-Wi symmetric state to a diverging "D" state where the fluid avoids the gap between the cylinders and flows symmetrically around their sides. The velocity field is qualitatively similar to that for viscoelastic flow around a single obstacle $[7,40]$. Further increasing $\mathrm{Wi}$, the system undergoes a second transition at $\mathrm{Wi}_{2} \approx 50$ to an asymmetric-diverging "AD" state in which the fluid selects a single preferred path either above $[y>0]$ or below $[y<0]$ the pair of cylinders [see Fig. 2(c)]. This randomly chosen bias is also similar to that observed for viscoelastic shear-thinning fluids flowing around a single cylinder [7,8,37].

For large $G=0.603$, the first transition at $\mathrm{Wi}_{1}$ results in a converging " $\mathrm{C}$ " flow state where the fluid flows preferentially between the cylinders, avoiding the gaps at their sides [Fig. 2(e)]. In contrast to the small- $G$ case, as Wi increases there is no second transition at $\mathrm{Wi}_{2}$ and the $\mathrm{C}$ state is maintained until the flow eventually becomes time dependent at $\mathrm{Wi} \gg \mathrm{Wi}_{1}$. The nature of the time 

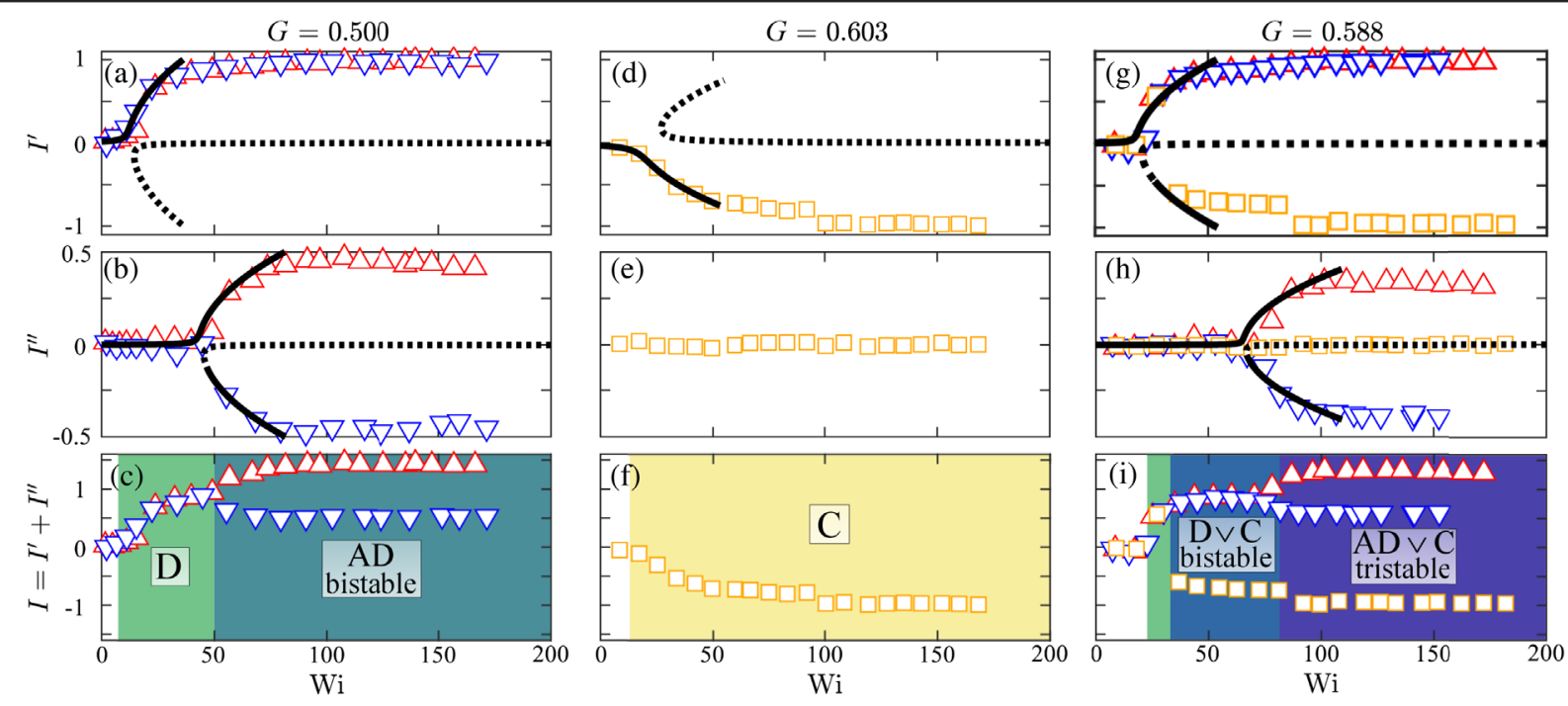

FIG. 3. Flow asymmetry parameters $I^{\prime}, I^{\prime \prime}$, and $I=I^{\prime}+I^{\prime \prime}$ versus Wi for microfluidic channels with (a)-(c) $G=0.500$, (d)-(f) $G=0.603$, and (g)-(i) $G=0.588$. Symbols indicate the qualitative flow states observed at high Wi for a given experiment: AD state with $I^{\prime \prime}>0$ (up pointing triangles) or $I^{\prime \prime}<0$ (down pointing triangles), and $\mathrm{C}$ state (squares). The solid and dotted black curves are fits of a fourth-order Landau potential to the data (see main text). Colored backgrounds in (c),(f), and (i) delineate the various flow states.

dependence is qualitatively similar to that previously reported for a single cylinder [7] and will not be discussed further here. For single isolated cylinders, the flow destabilizes due to the accumulation of elastic tensile stress along the curved streamlines close to the downstream stagnation point $[28,29,37]$, resulting in a small but finite random sideways perturbation of the downstream wake. If elasticity in the wake and shear thinning at the sides of the cylinder are sufficient, a positive feedback mechanism results in the growth of a steady flow asymmetry with random handedness and described as a supercritical pitchfork bifurcation [8,37]. In the present case, the bifurcations at each of the side-by-side cylinders at $\mathrm{Wi}_{1}$ are of opposite handedness, giving rise to the flow patterns shown in Figs. 2(b) and 2(e). This is due to the disparity in characteristic shear rate between the intercylinder and the cylinder-wall gaps. We note that a shear-thinning, but non-shear-banding viscoelastic polymer solution shows analogous flow behavior to that seen in Fig. 2 (Figs. S2 and S3 [45]).

We quantify the critical flow behavior using two dimensionless flow asymmetry parameters $I^{\prime}$ and $I^{\prime \prime}$ :

$I^{\prime}=\frac{\frac{1}{2}\left(\bar{u}_{+}+\bar{u}_{-}\right)-\bar{u}_{0}}{\frac{1}{2}\left(\bar{u}_{+}+\bar{u}_{-}\right)+\bar{u}_{0}} \quad$ and $\quad I^{\prime \prime}=\frac{\bar{u}_{+}-\bar{u}_{-}}{\bar{u}_{+}+\bar{u}_{-}+\bar{u}_{0}}$.

Here, $\bar{u}_{+}, \bar{u}_{-}$, and $\bar{u}_{0}$ are the average values of $u$ in the upper, lower, and intercylinder gaps, respectively (see Fig. 1). $I^{\prime}$ serves as the order parameter to quantify the first transition from the low-Wi symmetric state to either the D or C states [Figs. 2(b) and 2(e)]. $I^{\prime}=0$ when the average flow through the upper and lower gaps equals the flow through the center. Transition to the D state results in $I^{\prime}>0$, since $\bar{u}_{0}$ decreases. Transition to the $\mathrm{C}$ state results in $I^{\prime}<0$, since $\bar{u}_{0}$ increases. $I^{\prime \prime}$ serves as the order parameter to quantify the second transition between the $\mathrm{D}$ and the AD states. $I^{\prime \prime}=0$ in the $\mathrm{D}$ state, since $\bar{u}_{+}=\bar{u}_{-}$ [Fig. 2(b)]. In the AD state, fluid flows preferentially through either the upper or lower gap [Fig. $2(c)$ ], resulting in $I^{\prime \prime}>0$ or $I^{\prime \prime}<0$, respectively.

The asymmetry parameters $I^{\prime}, I^{\prime \prime}$ are shown versus Wi in Fig. 3 for various values of $G$ and are fitted with a quartic (double-well) Landau-type potential minimized as

$$
\mathrm{Wi}=\mathrm{Wi}_{c}\left(g \epsilon^{2}+h \epsilon^{-1}+1\right),
$$

where $\mathrm{Wi}_{c}=\mathrm{Wi}_{1}$ or $\mathrm{Wi}_{2}$ is the critical Weissenberg number for the bifurcation, and the order parameter $\epsilon=$ $I^{\prime}$ or $I^{\prime \prime}$, respectively. In all the fits, the growth rate coefficient $g$ is order unity, and the asymmetric term in $h$ quantifies system imperfections that bias a transition to a favored branch. The phenomenological Landau model for equilibrium phase transitions has long been found to provide a good description of bifurcation phenomena in driven nonequilibrium systems including Newtonian and viscoelastic flows [5,7,51,52]. Equation (2) describes imperfect forward (supercritical) pitchfork bifurcations without hysteresis.

For small $G=0.500$, the first transition in $I^{\prime}$ [Fig. 3(a)] occurs at $\mathrm{Wi}_{1} \approx 13$, and is a slightly imperfect $(h \approx-0.016$ ) supercritical pitchfork bifurcation where the favored branch $\left(I^{\prime}>0\right)$ gives diverging (D) flow. The unfavored $\left(I^{\prime}<0\right)$ branch was never observed, but its hypothetical existence is indicated in Fig. 3(a) by the dotted line. With increasing 
$\mathrm{Wi}, I^{\prime} \rightarrow 1$, implying that almost no fluid passes between the cylinders [as qualitatively evident from Fig. 2(b)]. The second transition in $I^{\prime \prime}$ [Fig. 3(b)] from the D state to the asymmetric diverging (AD) state occurs at $\mathrm{Wi}_{2} \approx 44$. The imperfection in this second bifurcation is very small $(h \approx-0.0013)$. The favored branch gives $I^{\prime \prime}>0$, but the unfavored $I^{\prime \prime}<0$ branch can also be reached and followed by initiating the flow at a high Wi and subsequent quasistatic reduction. The complete bifurcation diagram showing the total asymmetry $I=I^{\prime}+I^{\prime \prime}$ versus Wi for $G=0.500$ is shown in Fig. 3(c). The first bifurcation results in $I \rightarrow 1$. The second bifurcation splits $I$ into two branches, $I \rightarrow 1.5$ or $I \rightarrow 0.5$.

The behavior for $G=0.603$ is shown in Figs. 3(d)-3(f). In this case, the first bifurcation occurs at $\mathrm{Wi}_{1} \approx 20$ [Fig. 3(d)]. The asymmetric term in Eq. (2) is positive $(h \approx 0.033)$, resulting in a preferred transition from symmetric to converging (C) flow. With increasing Wi, $I^{\prime} \rightarrow-1$, indicating that nearly all of the fluid passes between the cylinders [see Figs. 2(e) and 2(f)]. Since $h$ is relatively large, the negative $I^{\prime}$ branch is strongly preferred. The positive $I^{\prime}$ branch [dotted line in Fig. 3(d)] is never observed experimentally. When the system selects the $\mathrm{C}$ state at the first transition, a second bifurcation is not observed, and $I^{\prime \prime} \approx 0$ for all Wi [Fig. 3(e)]. The complete bifurcation diagram for $G=0.603$ is shown in Fig. 3(f), since $I^{\prime \prime} \approx 0, I \approx I^{\prime}$.

The data shown in Figs. 2, 3(a)-3(f) demonstrate two disparate flow behaviors that are sensitive to the value of $G$. The first bifurcation to either the D or C states is well described as a supercritical pitchfork bifurcation quantified by $I^{\prime}$. The two states are different branches of the same bifurcation and the value of $G$ determines which branch is selected by changing the sign of the asymmetric term $(h)$ in Eq. (2). This implies the existence of a specific intermediate value of $G$ at which the bifurcation of $I^{\prime}$ should be perfect $(h=0)$ and the D or C states are equally likely.

By examining a range of intermediate values $0.56<G<$ 0.60 , we confirmed this assumption, as exemplified by Figs. 3(g)-3(i) for $G=0.588$. Here, increasing Wi quasistatically from 0 , the favored positive $I^{\prime}$ branch (D state) is observed [Fig. 3(g), triangles] on exceeding $\mathrm{Wi}_{1} \approx 20$. However, the imperfection is sufficiently small $(h \approx-0.007)$, that by quasistatic reduction of Wi from a high value, the unfavored $\mathrm{C}$ state branch (squares) can also be followed. From the $\mathrm{D}$ state, on exceeding $\mathrm{Wi}_{2} \approx 66$, the second bifurcation to the $\mathrm{AD}$ state occurs to either positive or negative $I^{\prime \prime}$ with almost equal likelihood in a given experiment [Fig. 3(h), triangles] since $h \approx 0$. The complete bifurcation diagram for $G=0.588$ in Fig. 3(i) shows the bistable coexistence of the $\mathrm{C}$ and $\mathrm{D}$ states for $\mathrm{Wi}_{1}<\mathrm{Wi}<\mathrm{Wi}_{2}$. For $\mathrm{Wi}>\mathrm{Wi}_{2}$, the system is tristable, where the two $\mathrm{AD}$ branches and the $\mathrm{C}$ branch coexist.

The behavior observed in all 11 microchannels is summarized in a flow stability diagram in $\mathrm{Wi}-G$ state

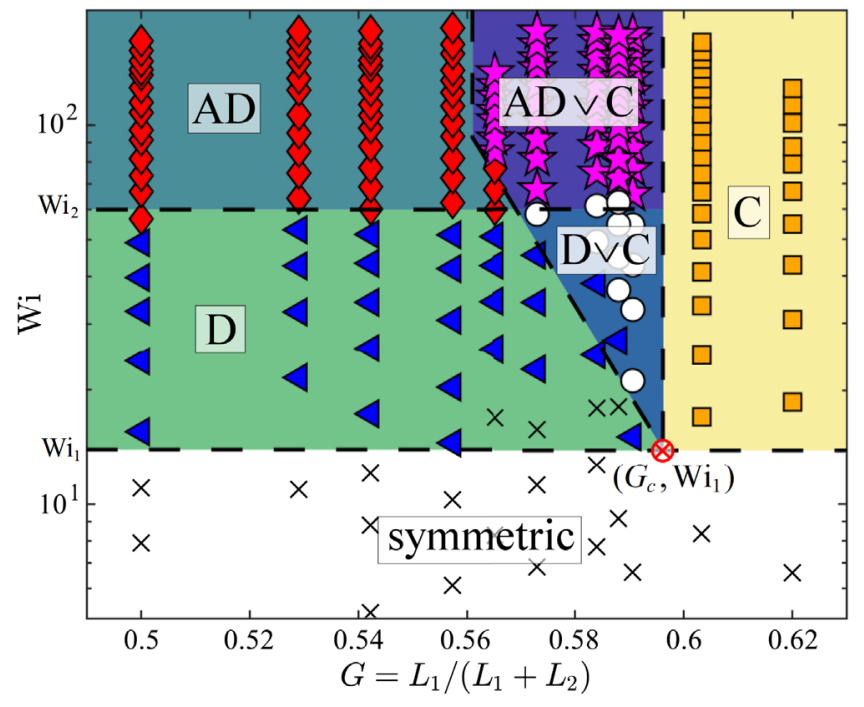

FIG. 4. Flow stability diagram in $\mathrm{Wi}-G$ state space. The dashed lines and colored shades delineate between flow states.

space (Fig. 4), where the plotted flow states and boundaries were determined directly from the measured velocity fields. For channels with small $G \lesssim 0.560$, the systems behave as exemplified by Figs. 2(a)-2(c), 3(a)-3(c), with a first bifurcation at $\mathrm{Wi}_{1}$ that is biased to the $\mathrm{D}$ state and a second bifurcation at $\mathrm{Wi}_{2}$ to the bistable $\mathrm{AD}$ state with either positive or negative $I^{\prime \prime}$. For large $G \gtrsim 0.595$, the systems behave as shown by Figs. 2(d)-2(f), 3(d)-3(f); the bifurcation at $\mathrm{Wi}_{1}$ is biased to the $\mathrm{C}$ state (which is maintained until the onset of time dependence at $\mathrm{Wi} \gg \mathrm{Wi}_{1}$ ). For a narrow range of $0.560 \lesssim G \lesssim 0.595$, beyond $\mathrm{Wi}_{1}$ it is possible to pass through a region of bistability between the $\mathrm{D}$ and $\mathrm{C}$ states before entering a tristable region beyond $\mathrm{Wi}_{2}$ comprising the bistable $\mathrm{AD}$ state and the $\mathrm{C}$ state. It is assumed that the $\mathrm{D} \vee \mathrm{C}$ bistable region meets the low-Wi symmetric region at a hypothetical single point $\left(G_{c}, \mathrm{Wi}_{1}\right)$ in the state space (red crossed circle, Fig. 4) where the first bifurcation would be perfect $(h=0)$. To the left of this point, the diagonal boundary between the $\mathrm{D} \vee \mathrm{C}$ and $\mathrm{D}$ regions reflects the increasing imperfection of the first bifurcation with decreasing $G$. However, based on our experiments, to the right of $G_{c}$ the boundary between the $\mathrm{D} \vee \mathrm{C}$ and the $\mathrm{C}$ states appears to be extremely abrupt. A small increase in $G>G_{c}$ causes a significant bias to the C state in the first bifurcation. A special case arises for $G=0.565$, where the tristable $\mathrm{AD} \vee \mathrm{C}$ region is reached by passing through the bistable $\mathrm{AD}$ region, avoiding the bistable $\mathrm{D} \vee \mathrm{C}$ region. Apparently, at this value of $G$ and $\mathrm{Wi}$, the selected flow path can spontaneously switch from an edge to the center gap, and vice versa for decreasing Wi.

By varying the geometric parameter space, we have demonstrated the complex dynamical behavior for the flow of a model Maxwell-type viscoelastic fluid past side-byside microcylinders. Although the flow states we report appear to be rather general for shear-thinning viscoelastic 
fluids $[8,45]$, we note that the rheological parameters of the test fluid (shear and extensional viscosity and relaxation time) are expected to influence the intensity of the flow asymmetry, the critical Wi values, and also the detailed form of the phase diagram. A proper comparison between different model rheological fluids will be instructive and enlightening.

There is a growing body of literature revealing important inertialess bifurcations to bistable flow states in microfluidic circuits (see, e.g., Refs. [53-55]), including the supercritical transitions in viscoelastic flows through cross slots $[3,4]$ and around single cylinders [7,37]. Here, despite the modest increase in geometrical complexity from a single cylinder, the dynamical behavior is significantly richer and shows how a combination of bifurcations can lead to multistability. Our results suggest a new interpretation for how viscoelastic fluids select preferred flow paths through ordered and disordered porous arrays (see, e.g., Ref. [17]), indicating that each individual obstacle should be considered as a bifurcation point, which can be perfect (ordered) or imperfect (disordered), depending on the spacing between nearest neighbor array elements.

We gratefully acknowledge the support of the Okinawa Institute of Science and Technology Graduate University (OIST) with subsidy funding from the Cabinet Office, Government of Japan, and also funding from the Japan Society for the Promotion of Science (JSPS, Grants No. 18K03958, No. 18H01135, and No. 20K14656) and the Joint Research Projects (JRPs) supported by the JSPS and the Swiss National Science Foundation (SNSF).

[1] H. A. Stone, A. D. Stroock, and A. Ajdari, Annu. Rev. Fluid Mech. 36, 381 (2004).

[2] T. M. Squires and S. R. Quake, Rev. Mod. Phys. 77, 977 (2005).

[3] P. E. Arratia, C. C. Thomas, J. Diorio, and J. P. Gollub, Phys. Rev. Lett. 96, 144502 (2006).

[4] R. J. Poole, M. A. Alves, and P. J. Oliveira, Phys. Rev. Lett. 99, 164503 (2007).

[5] N. Burshtein, K. Zografos, A. Q. Shen, R. J. Poole, and S. J. Haward, Phys. Rev. X 7, 041039 (2017).

[6] A. A. Dey, Y. Modarres-Sadeghi, and J. P. Rothstein, Phys. Rev. Fluids 3, 063301 (2018).

[7] S. J. Haward, N. Kitajima, K. Toda-Peters, T. Takahashi, and A. Q. Shen, Soft Matter 15, 1927 (2019).

[8] S. J. Haward, C. C. Hopkins, and A. Q. Shen, J. NonNewtonian Fluid Mech. 278, 104250 (2020).

[9] C. C. Hopkins, S. J. Haward, and A. Q. Shen, Small 16, 1903872 (2020).

[10] S. Varchanis, A. Syrakos, Y. Dimakopoulos, and J. Tsamopoulos, J. Non-Newtonian Fluid Mech. 267, 78 (2019).

[11] J. Zilz, C. Schäfer, C. Wagner, R. J. Poole, M. A. Alves, and A. Lidner, Lab Chip 14, 351 (2014).
[12] M.-N. Wei, B. Li, R. L. A. David, S. C. Jones, V. Sarohia, J. A. Schmitigal, and J.A. Kornfield, Science 350, 72 (2015).

[13] B. Keshavarz, E. C. Houze, J. R. Moore, M. R. Koerner, and G. H. McKinley, Phys. Rev. Lett. 117, 154502 (2016).

[14] M. Brust, C. Schaefer, R. Doerr, L. Pan, M. Garcia, P. E. Arratia, and C. Wagner, Phys. Rev. Lett. 110, 078305 (2013).

[15] M. Thiébaud, Z. Shen, J. Harting, and C. Misbah, Phys. Rev. Lett. 112, 238304 (2014).

[16] C. A. Browne, A. Shih, and S. S. Datta, Small 16, 1903944 (2020).

[17] D. M. Walkama, N. Waisbord, and J. S. Guasto, Phys. Rev. Lett. 124, 164501 (2020).

[18] M. Müller, J. Vorwerk, and P. O. Brunn, Rheol. Acta 37, 189 (1998).

[19] U. Eberhard, H. J. Seybold, E. Secchi, J. Jiménez-Martínez, P. A. Rühs, A. Ofner, J. S. Andrade, Jr., and M. Holzne, Sci. Rep. 10, 11733 (2020).

[20] S. De, J. van der Schaaf, N. G. Deen, J. A. M. Kuipers, E. A. J. F. Peters, and J. T. Padding, Phys. Fluids 29, 113102 (2017).

[21] D. Kawale, E. Marques, P. L. J. Zitha, M. T. Kreutzer, W. R. Rossen, and P. E. Boukany, Soft Matter 13, 765 (2017).

[22] E. M. Ekanem, S. Berg, S. De, A. Fadili, T. Bultreys, M. Rücker, J. Southwick, J. Crawshaw, and P. F. Luckham, Phys. Rev. E 101, 042605 (2020).

[23] G. Chauveteau and M. Moan, J. Phys. (Paris), Lett. 42, 201 (1981).

[24] R. Haas and F. Durst, Rheol. Acta 21, 566 (1982).

[25] N. E. Dyakonova, J. A. Odell, Y. V. Brestkin, A. V. Lyulin, and A. E. Saez, J. Non-Newtonian Fluid Mech. 67, 285 (1996).

[26] S. J. Haward and J. A. Odell, Rheol. Acta 42, 516 (2003).

[27] A. Anbari, H.-T. Chien, S. S. Datta, W. Deng, D. A. Weitz, and J. Fan, Small 14, 1703575 (2018).

[28] P. Pakdel and G. H. McKinley, Phys. Rev. Lett. 77, 2459 (1996).

[29] G. H. McKinley, P. Pakdel, and A. Öztekin, J. NonNewtonian Fluid Mech. 67, 19 (1996).

[30] A. N. Morozov and W. van Saarloos, Phys. Rep. 447, 112 (2007).

[31] L. Pan, A. Morozov, C. Wagner, and P. E. Arratia, Phys. Rev. Lett. 110, 174502 (2013).

[32] B. Qin and P.E. Arratia, Phys. Rev. Fluids 2, 083302 (2017).

[33] A. Groisman and V. Steinberg, Nature (London) 405, 53 (2000).

[34] A. N. Morozov and W. van Saarloos, Phys. Rev. Lett. 95, 024501 (2005).

[35] D. Bonn, F. Ingremeau, Y. Amarouchene, and H. Kellay, Phys. Rev. E 84, 045301(R) (2011).

[36] J. Page, Y. Dubief, and R. R. Kerswell, Phys. Rev. Lett. 125, 154501 (2020).

[37] S. Varchanis, C. C. Hopkins, A. Q. Shen, J. Tsamopoulos, and S. J. Haward, Phys. Fluids 32, 053103 (2020).

[38] X. Shi and G. F. Christopher, Phys. Fluids 28, 124102 (2016).

[39] A. Varshney and V. Steinberg, Phys. Rev. Fluids 2, 051301(R) (2017). 
[40] S. J. Haward, K. Toda-Peters, and A. Q. Shen, J. NonNewtonian Fluid Mech. 254, 23 (2018).

[41] S. Peng, Y.-L. Xiong, X.-Y. Xu, and P. Yu, Phys. Fluids 32, 083106 (2020).

[42] H. Rehage and H. Hoffmann, J. Phys. Chem. 92, 4712 (1988).

[43] H. Rehage and H. Hoffmann, Mol. Phys. 74, 933 (1991).

[44] S. M. Fielding, J. Rheol. 60, 821 (2016).

[45] See Supplemental Material at http://link.aps.org/ supplemental/10.1103/PhysRevLett.126.054501 for the rheological characterization of the WLM solution and cursory flow experiments using a shear-thinning polymer solution, which includes Refs. [46,47]

[46] R. B. Bird, R. C. Armstrong, and O. Hassager, Dynamics of Polymeric Liquids (John Wiley and Sons, New York, 1987).

[47] S. L. Anna and G. H. McKinley, J. Rheol. 45, 115 (2001).
[48] N. Burshtein, S. T. Chan, K. Toda-Peters, A. Q. Shen, and S. J. Haward, Curr. Opin. Colloid Interface Sci. 43, 1 (2019).

[49] S. T. Wereley and C. D. Meinhart, in Microscale Diagnostic Techniques, edited by K. S. Breuer (Springer, Berlin, 2005), pp. 51-112.

[50] S. T. Wereley and C. D. Meinhart, Annu. Rev. Fluid Mech. 42, 557 (2010).

[51] A. Aitta, G. Ahlers, and D. S. Cannell, Phys. Rev. Lett. 54, 673 (1985).

[52] J. P. Gollub and M. H. Freilich, Phys. Fluids 19, 618 (1976).

[53] A. Groisman, M. Enzelberger, and S. R. Quake, Science 300, 955 (2003).

[54] J. B. Geddes, B. D. Storey, D. Gardner, and R. T. Carr, Phys. Rev. E 81, 046316 (2010).

[55] C. A. Browne, A. Shih, and S. S. Datta, J. Fluid Mech. 890, A2 (2020). 